\title{
Broadband transmission-line illusions based on transformation electromagnetic
}

\author{
Tsutomu Nagayama ${ }^{1}$ and Atsushi Sanada ${ }^{2, *}$ \\ ${ }^{1}$ Graduate School of Science and Engineering, Kagoshima University, Kagoshima 890-0065, Japan \\ ${ }^{2}$ Graduate School of Engineering Science, Osaka University, Osaka 560-8531, Japan
}

Received: 30 August 2018 / Accepted: 11 October 2019

\begin{abstract}
We demonstrate broadband transmission-line illusions based on transformation electromagnetics at microwave frequencies by using the distributed full-tensor anisotropic medium. Due to an intrinsic nature of the non-resonant unit cell of the medium, the illusions operate from DC to an upper limit frequency where the homogeneous medium approximation holds. Two-dimensional groove and bump illusion media mimicking scattered waves by an original groove and a bump are designed. Their broadband and incident angle independent operations are confirmed by circuit simulations. The groove illusion medium is implemented on a dielectric substrate with microstrip-line technology, and it is confirmed experimentally by near-field measurements that the illusion medium well mimics scattered waves by the original groove in the broadband frequency range from $2.60 \mathrm{GHz}$ to $4.65 \mathrm{GHz}$.
\end{abstract}

Keywords: Electromagnetic illusion / transformation electromagnetics / metamaterials

\section{Introduction}

The distributed full-tensor anisotropic medium [1] has been proposed. The medium consists of a two-dimensional (2-D) array of unit cells each of which is a distributed transmission-line network yielding equivalent full-tensor material parameters [1]. According to the equivalent circuit analysis of the medium, it is directly shown that the diagonal components of the permeability tensor correspond to inductances per unit length in the $x$ - and $y$-branches and the off-diagonal components correspond to the mutual inductance per unit length between the $x$ - and $y$-branches. With these intuitive properties, the medium has full controllability of the material tensor parameters and is suitable for implementations of cloaks of invisibility [1-14] based on the concept of transformation electromagnetics [15-17]. In addition, the medium is advantageous in wideband operations from DC to an upper limit frequency where the homogeneous medium approximation holds due to an intrinsic nature of the non-resonant unit cells [1]. With the full-tensor anisotropic medium, a carpet cloak of invisibility [1] and coordinate transformation resonators [18] have been implemented and their unique operations have been demonstrated.

Due to the flexibility of the design, the medium can be used not only for suppressing scattered waves to hide an object or mimicking a shape of other resonator but

\footnotetext{
* e-mail: sanada@ieee.org
}

also for mimicking scattered waves from a non-existent object to realize electromagnetic/optical illusions $[19,20]$. In this paper, we demonstrate broadband transmission-line illusions by using the distributed full-tensor anisotropic medium. In Section 2, we show the concept of groove and bump illusions and recall the theory of the transmission-line anisotropic media. In Section 3, the groove and bump illusion media are designed and transmission-line parameters are obtained. In Section 4, SPICE circuit simulations are carried out to confirm operations of the designed groove and bump illusions. In Section 5, the groove illusion medium is implemented on a dielectric substrate and the illusion operation is demonstrated by near-field measurements at microwave frequencies.

\section{Illusions with transmission-line anisotropic media}

\subsection{Groove and bump illusions}

Here, we consider two types of illusions: a groove illusion and a bump illusion. The groove illusion mimicking scattering waves by a 2-D groove on a flat surface whose cross section is shown in Figure 1a. The illusion medium of Figure $1 \mathrm{~b}$ is designed to generate identical scattered waves by the groove. In addition, the bump illusion mimicking the scattering waves by a $2-\mathrm{D}$ bump on a flat surface is designed with the same manner (see Fig. 2). 


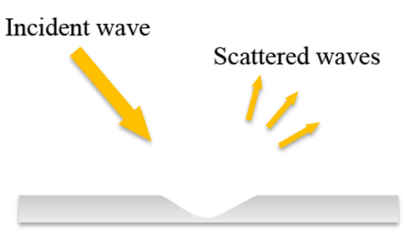

(a)

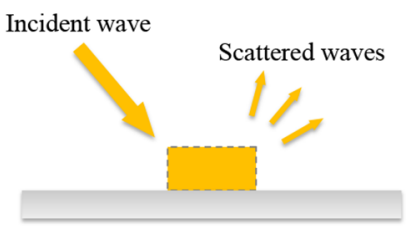

(b)
Fig. 1. Groove illusion. (a) Scattered waves by a groove to be mimicked. (b) Scattered waves by an illusion medium on a flat surface.

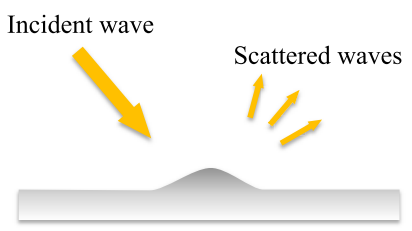

(a)

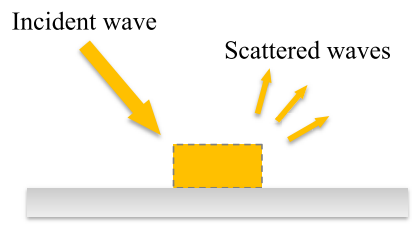

(b)
Fig. 2. Bump illusion. (a) Scattered waves by a bump to be mimicked. (b) Scattered waves by an illusion medium on a flat surface.

For both cases, the illusions can be realized with inhomogeneous anisotropic media equivalent to a corresponding coordinate transformation from an original Cartesian coordinate system $(x, y)$ into a non-conformal coordinate system $\left(x^{\prime}, y^{\prime}\right)$. According to the material interpretation of the coordinate transformation [15-17], the material tensor parameters are given by

$$
\mu^{i^{\prime} j^{\prime}}=\left|\operatorname{det}\left(g^{i^{\prime} j^{\prime}}\right)\right|^{-1 / 2} g^{i^{\prime} j^{\prime}} \mu, \quad \varepsilon^{i^{\prime} j^{\prime}}=\left|\operatorname{det}\left(g^{i^{\prime} j^{\prime}}\right)\right|^{-1 / 2} g^{i^{\prime} j^{\prime}} \varepsilon
$$

where $g^{i^{\prime} j^{\prime}}$ is the metric for the coordinate transformation.

\subsection{Broadband transmission-line illusion media}

The groove and bump illusions are realized by using the distributed transmission-line full-tensor anisotropic medium shown in Figure 3 [1]. The characteristic impedances and electrical lengths of the transmission-line sections in the unit cell are directly determined from the material tensor parameters of equation (1) by [1]:

$$
\begin{aligned}
& \mu_{y y}-\mu_{x y}=\frac{2 Z_{0 x}}{\omega \Delta d} \tan (\beta l / 2), \\
& \mu_{x x}-\mu_{x y}=\frac{2 Z_{0 y}}{\omega \Delta d} \tan (\beta l / 2), \\
\mu_{x y}=\mu_{y x}= & \pm 2\left[Z_{0 \mathrm{M}}^{2}\left(Y_{0 x}+Y_{0 y}\right) \tan \left(\frac{\beta l}{2}\right)\right. \\
& \left.+Z_{0 \mathrm{M}}\left\{\operatorname{cosec}\left(\beta_{\mathrm{M}} l_{\mathrm{M}}\right)-\cot \left(\beta_{\mathrm{M}} l_{\mathrm{M}}\right)\right\}\right] \\
& {\left[\omega \Delta d \left\{\cos ^{2}\left(\frac{\beta l}{2}\right)\right.\right.} \\
& +Z_{0 \mathrm{M}}\left(Y_{0 x}+Y_{0 y}\right) \sin (\beta l) \cot \left(\beta_{\mathrm{M}} l_{\mathrm{M}}\right) \\
& \left.\left.-Z_{0 \mathrm{M}}^{2}\left(Y_{0 x}+Y_{0 y}\right)^{2} \sin ^{2}\left(\frac{\beta l}{2}\right)\right\}\right],
\end{aligned}
$$

where $\left(L_{0 x}^{\prime}, C_{0 x}^{\prime}\right), \quad\left(L_{0 y}^{\prime}, C_{0 y}^{\prime}\right), \quad\left(L_{0 \mathrm{M}}^{\prime}, \quad C_{0 \mathrm{M}}^{\prime}\right)$ are the inductances per-unit-length and the capacitances per-

\section{Designs}

\subsection{Groove illusion} unit-length of the corresponding the transmission-line sections with the characteristic impedances of $Z_{0 x}, Z_{0 y}$, and $Z_{0 \mathrm{M}}$, respectively.

Fig. 3. Unit cells of the distributed full-tensor anisotropic medium. (a) $\mu_{x y}>0$. (b) $\mu_{x y}<0$.

$$
\begin{aligned}
\varepsilon_{z}= & \frac{1}{\omega \Delta d}\left\{\left(Y_{0 x}+Y_{0 y}\right) \sin (\beta l) \cos \left(\beta_{\mathrm{M}} l_{\mathrm{M}}\right)\right. \\
& +Y_{0 \mathrm{M}} \cos ^{2}\left(\frac{\beta l}{2}\right) \sin \left(\beta_{\mathrm{M}} l_{\mathrm{M}}\right) \\
& \left.-Z_{0 \mathrm{M}}\left(Y_{0 x}+Y_{0 y}\right)^{2} \sin ^{2}\left(\frac{\beta l}{2}\right) \sin \left(\beta_{\mathrm{M}} l_{\mathrm{M}}\right)\right\},
\end{aligned}
$$

where $\Delta d$ is the unit cell length and $\beta l=\beta_{x} l_{x}=\beta_{y} l_{y}$ are the electrical lengths of the transmission-lines in the $x$ - and $y$-branches in Figure 3.

It is worth mentioning that the medium essentially has extremely broadband characteristics. The broadband ature of this medium can be seen from the fact that, as the frequency approaches to zero, the material parameters become frequency independent [1] as:

$$
\begin{gathered}
\mu_{y y}-\mu_{x y} \rightarrow L_{0 x}^{\prime}, \\
\mu_{x x}-\mu_{x y} \rightarrow L_{0 y}^{\prime}, \\
\mu_{x y}=\mu_{y x} \rightarrow \pm L_{0 \mathrm{M}}^{\prime} \frac{C_{0 x}^{\prime}+C_{0 y}^{\prime}}{C_{0 x}^{\prime}+C_{0 y}^{\prime}+C_{0 \mathrm{M}}^{\prime}}, \\
\varepsilon_{z} \rightarrow C_{0 x}^{\prime}+C_{0 y}^{\prime}+C_{0 \mathrm{M}}^{\prime},
\end{gathered}
$$

Figure 4 shows the coordinate transformation $(x, y) \mapsto$ $\left(x^{\prime}, y^{\prime}\right)$ used in the groove illusion of Figure 1. The 


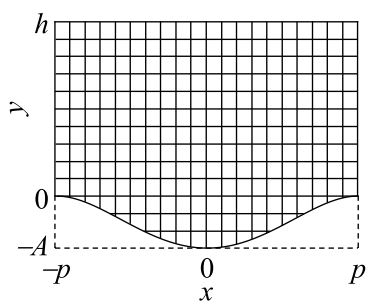

(a)

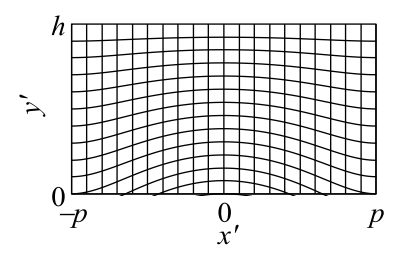

(b)
Fig. 4. Coordinate transformation for the groove illusion medium. (a) Original Cartesian coordinate system $(x, y)$. (b) Transformed nonconformal coordinate system $\left(x^{\prime}, y^{\prime}\right)$.

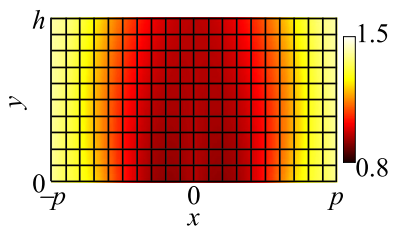

(a)

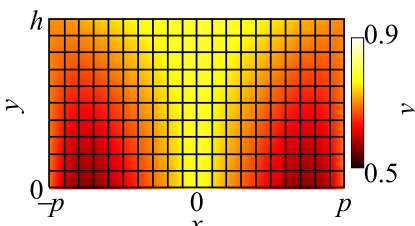

(c)

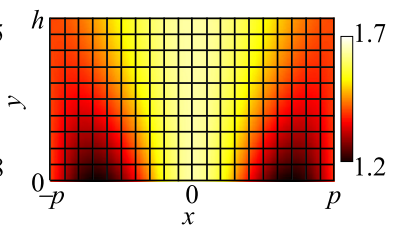

(b)

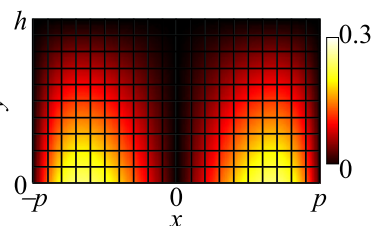

(d)
Fig. 5. Calculated characteristic impedances and electric lengths for the groove illusion of Figure 1. (a) Normalized characteristic impedance $Z_{0 x} / \eta$. (b) Normalized characteristic impedance $Z_{0 y} / \eta$. (c) Normalized electrical length $\beta l / k \Delta d$. (d) Normalized electrical length $\beta_{\mathrm{M}} l_{\mathrm{M}} / k \Delta d$. $\eta$ and $k$ are the wave impedance and the wave number in the original medium, respectively.

coordinate transformation is given by

$$
x^{\prime}=x, \quad y^{\prime}=\frac{y+A\left\{1-\left(\frac{x}{p}\right)^{2}\right\}^{2}}{h+A\left\{1-\left(\frac{x}{p}\right)^{2}\right\}^{2}} h,
$$

where $2 p$ is the width of the cloak and $A$ is the depth of the groove. The original and transformed regions are given with $-p \leq x \leq p,-A\left\{1-(x / p)^{2}\right\}^{2} \leq y \leq h,-p \leq x^{\prime} \leq p$, and $0 \leq y^{\prime} \leq h$.

Based on equation (10), the characteristic impedances and electrical lengths for each of the discretized unit cells are designed by solving equations (2) through (5) simultaneously with the designed material tensor parameters given by equation (1). In this design, we choose the depth and the width of the groove $A=0.3 h$ and $2 p=2 h$, respectively, where $h$ is the height of the groove illusion medium. The absolute refractive index $n=2.14$ and the wave impedance $\eta=63.6 \Omega$ of the original medium are also chosen in the following design. Figure 5 shows the calculated characteristic impedances and electrical lengths $Z_{0 x}, Z_{0 y}, \beta_{x} l_{x}=\beta_{y} l_{y} \equiv \beta l$, and $\beta_{\mathrm{M}} l_{\mathrm{M}}$. Here, we choose $Z_{0 \mathrm{M}}=1.5 \eta$ where $\eta$ is the wave impedance in the original medium.

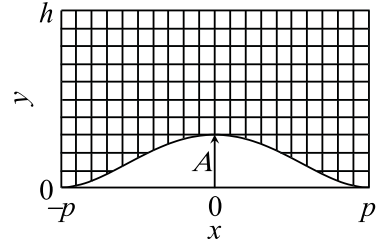

(a)

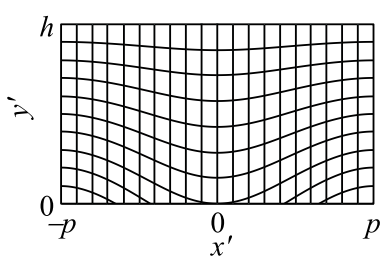

(b)
Fig. 6. Coordinate transformation for the bump illusion medium. (a) Original Cartesian coordinatesystem $(x, y)$. (b) Transformed nonconformal coordinate system $\left(x^{\prime}, y^{\prime}\right)$.

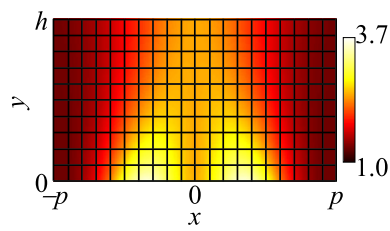

(a)

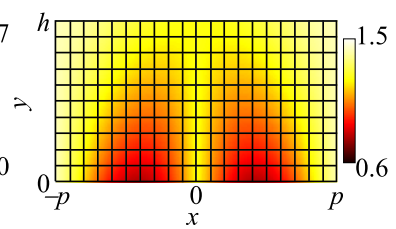

(b)

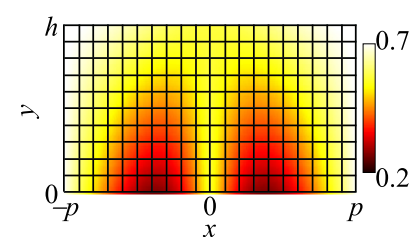

(c)

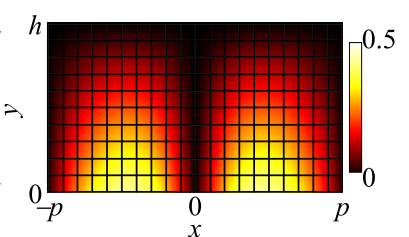

(d)
Fig. 7. Calculated characteristic impedances and electric lengths for the bump illusion of Figure 2. (a) Normalized characteristic impedance $Z_{0 x} / \eta$. (b) Normalized characteristic impedance $Z_{0 y} / \eta$. (c) Normalized electrical length $\beta l / k \Delta d$. (d) Normalized electrical length $\beta_{\mathrm{M}} l_{\mathrm{M}} / k \Delta d$.

\subsection{Bump illusion}

Figure 6 shows the coordinate transformation $(x, y) \mapsto$ $\left(x^{\prime}, y^{\prime}\right)$ used in the bump illusion of Figure 2 . In this case, we choose the coordinate transformation:

$$
x^{\prime}=x, \quad y^{\prime}=\frac{y-A\left\{1-\left(\frac{x}{p}\right)^{2}\right\}^{2}}{h-A\left\{1-\left(\frac{x}{p}\right)^{2}\right\}^{2}} h,
$$

where $2 p$ is the width of the illusion medium and $A$ is the height of the bump. In this case, the original and transformed regions are given with $-p \leq x \leq p, \quad A\{1-$ $\left.(x / p)^{2}\right\}^{2} \leq y \leq h,-p \leq x^{\prime} \leq p$, and $0 \leq y^{\prime} \leq h$.

Based on equation (11), the characteristic impedances and electrical lengths for each of the discretized unit cells are designed with the same manner as in the groove illusion. For the bump geometry with $A=0.3 h$ and $2 p=2 h$, where $h$ is the height of the illusion medium, the obtained characteristic impedances and electrical lengths $Z_{0 x}, Z_{0 y}$, $\beta_{x} l_{x}=\beta_{y} l_{y} \equiv \beta l$, and $\beta_{\mathrm{M}} l_{\mathrm{M}}$ are shown in Figure 7 . The values $n, \eta$, and $Z_{0 \mathrm{M}}$ are chosen as the same values for the case with the groove illusion. 


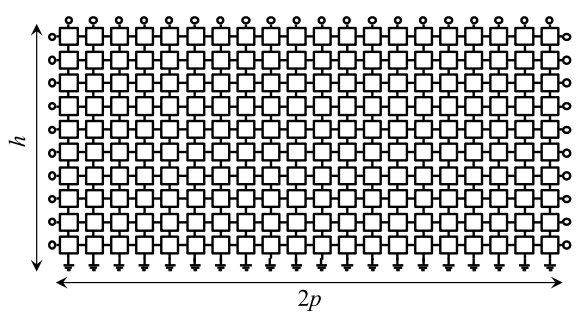

(a)

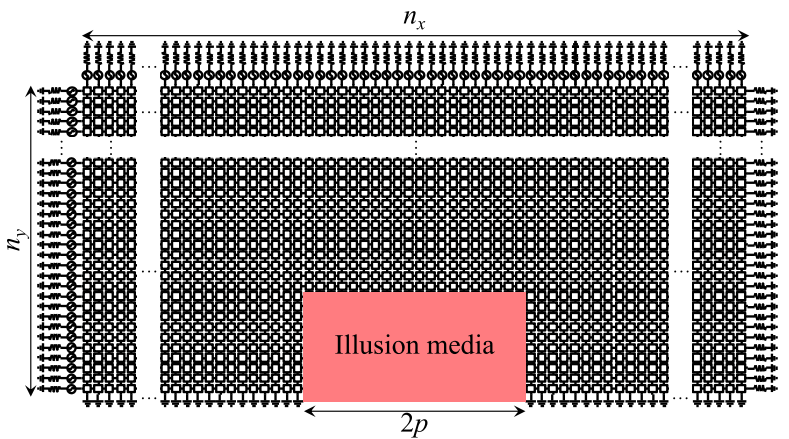

(b)

Fig. 8. Configurations of circuit simulations. (a) The groove or bump illusion medium area with $20 \times 10$ unit cells $(2 p \times h=$ $20 \Delta d \times 10 \Delta d$ ) (b) Calculation area with $n_{x} \times n_{y}=300 \times 150$ unit cells.

\section{Illusion simulations}

In order to show the equivalence of scattered waves by the groove and bump illusion media to those by the original obstacles as well as their broadband characteristics, wave scattering simulations are carried out using a SPICE simulator.

\subsection{Simulation setup}

Figure 8 shows the configuration of the circuit simulations. An illusion medium with the anisotropic transmission-line parameters of either Figure 5 or Figure 7 is discretized with $20 \times 10$ unit cells and is arranged at the bottom center of an isotropic medium of $300 \times 150$ unit cells [1]. The nodes on the bottom boundaries are short-circuited including the illusion area, and the nodes on the right-hand side boundary are terminated by resistors. Voltage sources are connected at the nodes on the other boundaries to illuminate the illusion medium with a normal or oblique Gaussian beams whose beam waist is $10 \Delta d$. Complex voltage distributions at the center nodes of the unit cells are calculated, and the results are compared to those with and without an original obstacle.

Here, the wave impedance and the refractive index in the isotropic area are chosen as $\eta=63.6 \Omega$ and $n=2.14$, respectively, considering feasibility of practical implementations. Sixty-two Ohm resistances are used for the terminations to simulate a practical situation used in the experiments. The internal impedance of each voltage source is also set to be $62 \Omega$.

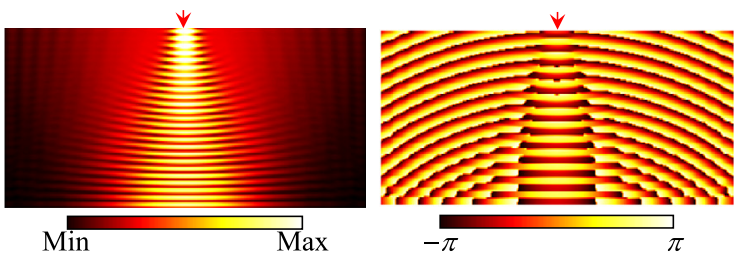

(a)

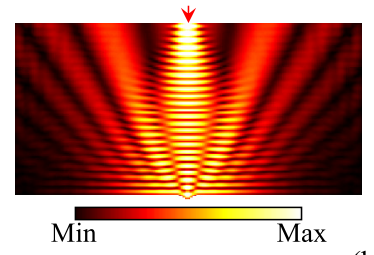

(b)
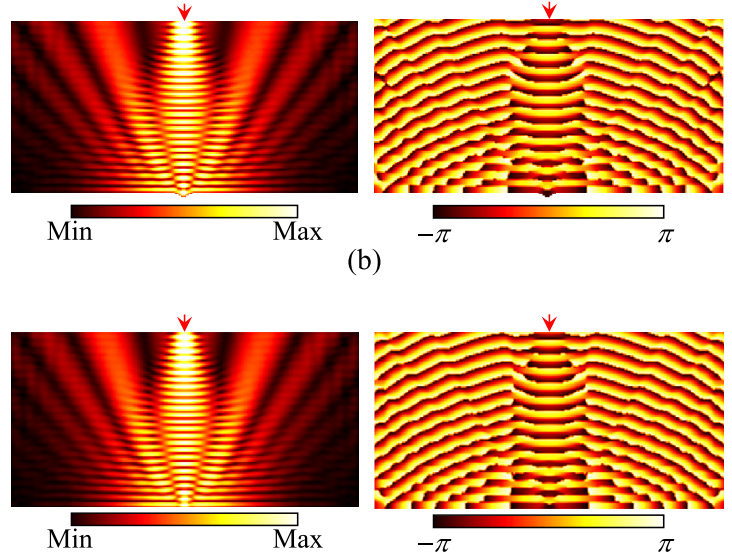

(c)

Fig. 9. Calculated complex voltage distributions for the case with the normal incident wave at the wavelength $\lambda_{g}=12 \Delta d$. Left: Amplitude. Right: Phase. (a) Flat surface. (b) With the original groove. (c) With the groove illusion medium.

\subsection{Groove illusion}

Figure 9 shows calculated voltage distributions with a normal incident wave of $\theta_{\text {inc }}=0$ deg for the cases with (a) a flat surface, (b) an original groove, and (c) the groove illusion medium. The wavelength is chosen as $\lambda_{g}=12 \Delta d$. As seen in Figure 9a, the incident wave is directly reflected back toward the source by the flat surface, whereas the incident wave is strongly scattered by the original groove in several directions as in Figure 9b. For the case with the groove illusion medium as in Figure 9c, the incident wave is scattered as if scattered by the original groove almost completely.

In order to quantitively evaluate the performance, the radar cross sections (RCSs) for the groove illusion medium are calculated and compared with those with the original groove. Figure 10 shows those calculated results. It is seen from the figure that the RCSs agree well with each other and the scattered waves are in the directions of $\theta_{\text {scat }}=0$, $\pm 27, \pm 43 \mathrm{deg}$, etc., where $\theta_{\text {scat }}$ is the reflection angle from the normal direction, which is consistent with the voltage distribution simulations in Figure 9. Therefore, it can be concluded that the illusion medium well mimics scattered waves by the original groove.

As mentioned in Section 2.2, the illusion medium intrinsically operates in an extremely broad frequency band down to DC. For instance, Figure 11a-c shows results for the case with the doubled wavelength of $\lambda_{g}=24 \Delta d$. In this case, the beam waist of the source is also doubled with $20 \Delta d$ to form a directive beam. It is seen from the figure that scattered waves by the designed illusion medium in 


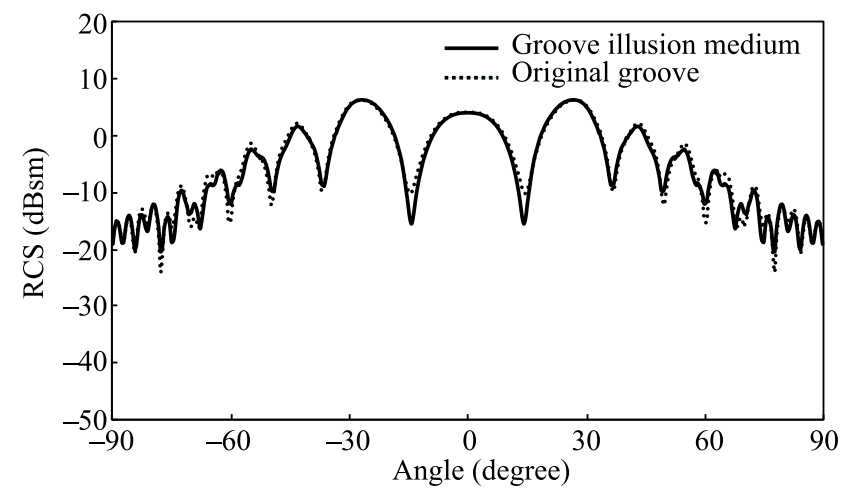

Fig. 10. Calculated RCSs for the groove illusion medium and the original groove.

Figure 11c almost completely agree with those by the original groove in Figure 11b. From the results of Figures 9 and 11, an octave bandwidth of the illusion medium operation is confirmed.

Moreover, simulations with oblique incident waves are carried out. Figure $12 \mathrm{a}-\mathrm{c}$ shows the results for the incident wave with the angle of (a) $30 \mathrm{deg}$, (b) $45 \mathrm{deg}$, and (c) $60 \mathrm{deg}$, respectively. The wavelength and the beam waist of the incident wave are chosen as $12 \Delta d$ and $10 \Delta d$, respectively. It is seen from the results that the groove illusion medium perfectly operates with these oblique incident angles. Thus, the incident angle independent operations of the groove illusion medium are confirmed.

\subsection{Bump illusion}

Figure 13 shows similar calculated results with a normal incident wave for the cases with (a) an original bump, and (b) the bump illusion medium. The wavelength and the beam waist of the incident wave are chosen to be the same as in the previous simulations, i.e., $12 \Delta d$ and $10 \Delta d$, respectively. It is seen from the figure that the bump illusion medium well mimics the scattered waves by the original bump. Figure 14 shows calculated RCSs for the bump illusion medium and the original bump. It is seen from the figure that both the RCSs agree well with each other.

Figure 15 also shows results for the cases with the oblique incident waves with (a) $30 \mathrm{deg}$, (b) $45 \mathrm{deg}$, and (c) $60 \mathrm{deg}$, respectively. It is seen from the figure that the bump illusion medium mimics the original bump well regardless of the oblique incident angles as in the case for the groove illusion.

\section{Groove illusion experiments}

The groove illusion medium designed in Section 3 is implemented with microstrip-line technology and its operation is confirmed by near-field measurements.

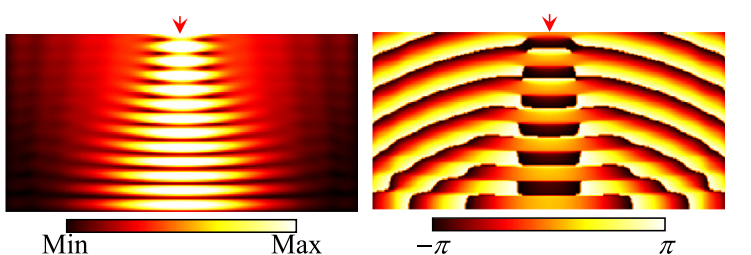

(a)

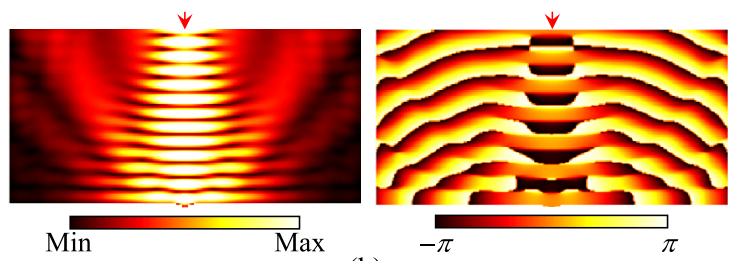

(b)

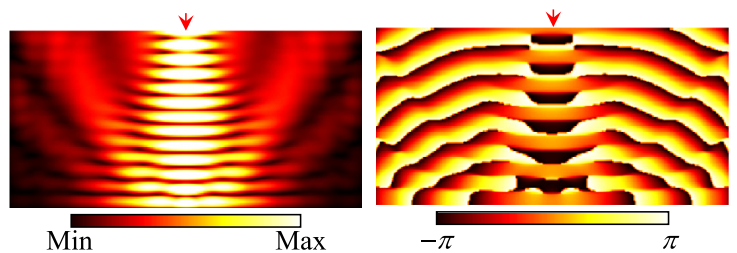

(c)

Fig. 11. Calculated complex voltage distributions for the case with the normal incident wave at the wavelength $\lambda_{g}=24 \Delta d$. Left: Amplitude. Right: Phase. (a) Flat surface. (b) With the original groove. (c) With the groove illusion medium.

\subsection{Implementation}

The groove illusion medium is implemented by using anisotropic unit cells of Figure 16 [1]. The unit cells are direct implementations of Figure 3 consisting of microstripline sections with line widths, $W_{x}, W_{y}$, and $W_{\mathrm{M}}$, and line lengths, $l_{x}, l_{y}$, and $l_{\mathrm{M}}$ (see Fig. 16) to satisfy the characteristic impedances and the electrical lengths given by equations (2)-(5). A dielectric substrate ARLON DiClad880 with the relative permittivity $\varepsilon_{r}=2.17$, the thickness $t=0.254 \mathrm{~mm}$, and the dielectric $\operatorname{loss} \tan \delta=$ 0.00085 is used. The width and the height of the illusion area are chosen as $2 p=100 \mathrm{~mm}$ and $h=50 \mathrm{~mm}$, respectively, and the unit element size is also chosen as $\Delta d=5 \mathrm{~mm}$. Figure 17 shows the line widths $W_{x}$ and $W_{y}$, and the line lengths $l_{x}=l_{y}=l$ and $l_{\mathrm{M}}$ calculated by Keysight ADS assuming the effective phase constant in the substrate $\beta=\beta_{x}=\beta_{y}=\beta_{\mathrm{M}}=1.316 k_{0}$, where $k_{0}$ is the wave number in vacuum. The width $W_{\mathrm{M}}$ is discretionally determined as $0.231 \mathrm{~mm}$ to give the value of $Z_{0 \mathrm{M}}=1.5 \eta$.

Figure 18a shows a photograph of the fabricated illusion medium. For comparison, an original medium with the groove shown in Figure 18b is also fabricated. Figure 18c is the magnified picture of the coordinate transformed area arranged on the bottom center of Figure 18a. The illusion area is surrounded by the homogeneous isotropic area of $280 \times 200 \mathrm{~mm}^{2}$. The nodes of the bottom boundary including the illusion area are short-circuited by throughhole vias with the diameter of $\phi=0.3 \mathrm{~mm}$, and nodes of the 

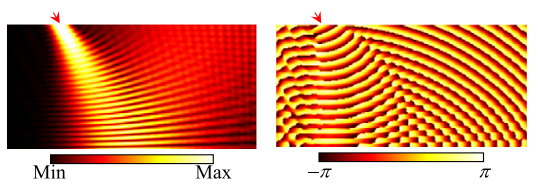

Flat surface
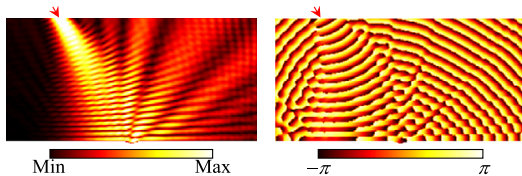

With the original groove
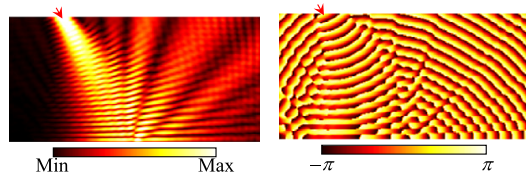

With the groove illusion medium

(a)
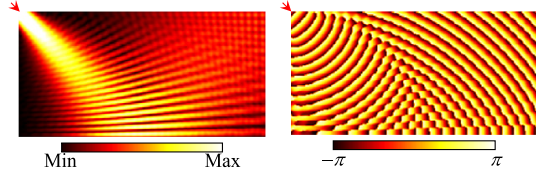

Flat surface
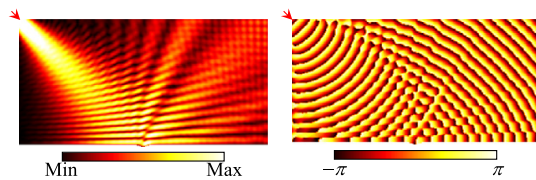

With the original groove
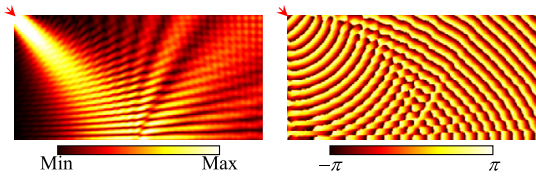

With the groove illusion medium

(b)
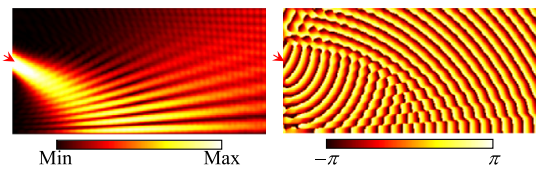

Flat surface
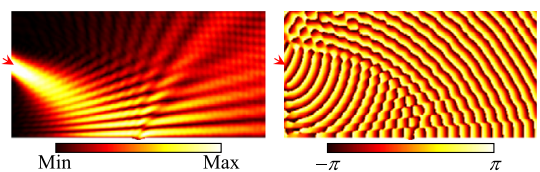

With the original groove
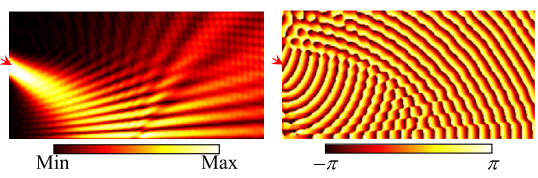

With the groove illusion medium

(c)

Fig. 12. Calculated complex voltage distributions for the cases with oblique incident waves at $\lambda_{q}=12 \Delta d$ (Top row: Flat surface. Center row: Original groove. Bottom row: Groove illusion medium). Left: Amplitude. Right: Phase. (a) $\theta_{\text {inc }}=30$ deg. (b) $\theta_{\text {inc }}=45$ deg. (c) $\theta_{\text {inc }}=60 \mathrm{deg}$.
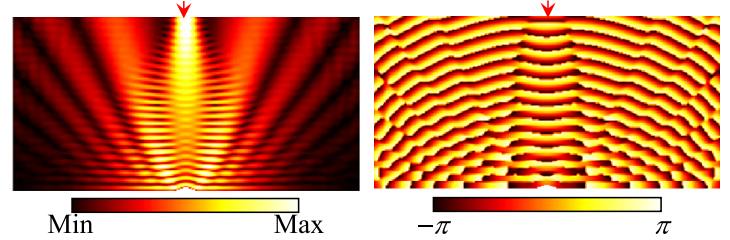

(a)
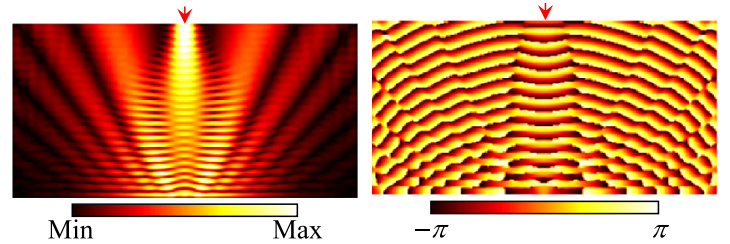

(b)

Fig. 13. Calculated complex voltage distributions for the case with the normal incident wave at the wavelength $\lambda_{g}=12 \Delta d$. Left: Amplitude. Right: Phase. (a) With the original bump. (b) With the bump illusion medium.

other boundaries are terminated by chip resistors with $62 \Omega$ thorough metallic lands with $0.8 \times 0.5 \mathrm{~mm}^{2}$ to prevent reflections.

\subsection{Near-field measurements}

\subsubsection{Field distributions with a source at the top center}

First, a coaxial cable for excitation is connected at the top center of the sample (Position A in Fig. 19), and near-field distributions $0.5 \mathrm{~mm}$ above the two samples are measured by a vector network analyzer using an electric probe with an automated $x y z$-stage at the sampling points with the period of $\Delta d / 4=1.25 \mathrm{~mm}$ (see the insets in Fig. 19 for the

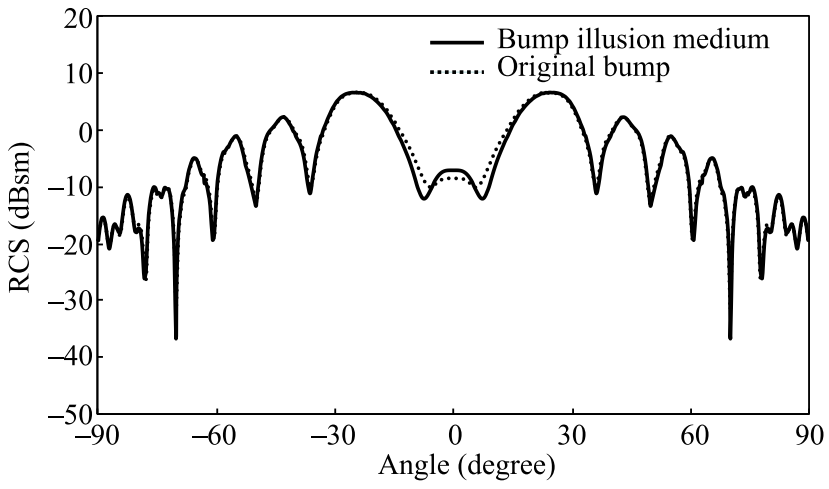

Fig. 14. Calculated RCSs for the bump illusion medium and the original bump.

electric probe and the excitation method). The length and radius of tip of the electric probe are $6.0 \mathrm{~mm}$ and $0.14 \mathrm{~mm}$, respectively.

Figure 20a and b shows measured complex electric near-field distributions for the cases with the groove illusion medium and with the original groove, respectively, at the highest frequency of $4.65 \mathrm{GHz}\left(\lambda_{g}=6.02 \Delta d\right)$ where illusion operations are observed in the experiments. It is seen from the figure that the groove illusion medium mimics the original groove well. For comparison, Figure 21a and b shows corresponding simulated complex voltage distributions for the cases with the simulated results with groove illusion medium and with the original groove, respectively, at the same frequency. By comparing Figure 20 with 21, it is seen that the measured field distributions agree well with the simulated predictions. Note that the major scattered waves are in \pm 29 deg 

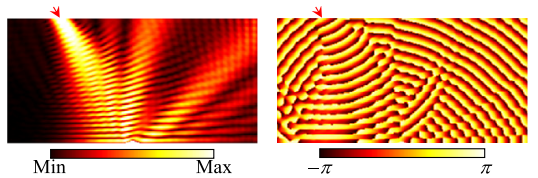

With the original bump
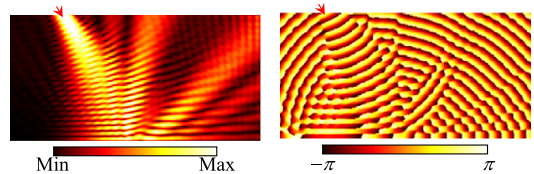

With the bump illusion medium

(a)

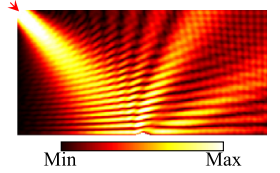

With the original bump

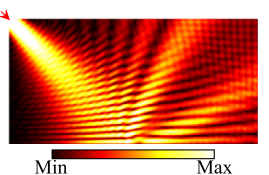

With the bump illusion medium

(b)
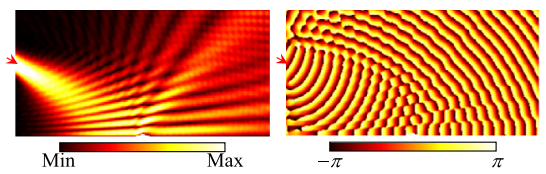

With the original bump

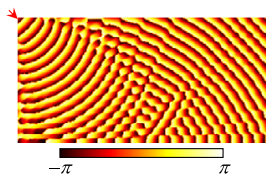

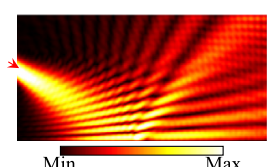

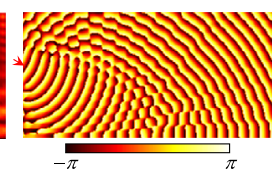

With the bump illusion medium

(c)

Fig. 15. Calculated complex voltage distributions for the cases with oblique incident waves at $\lambda_{g}=12 \Delta d$ (Top row: Original bump. Bottom row: Bump illusion medium). Left: Amplitude. Right: Phase. (a) $\theta_{\text {inc }}=30$ deg. (b) $\theta_{\text {inc }}=45$ deg. (c) $\theta_{\text {inc }}=60$ deg.

(a)

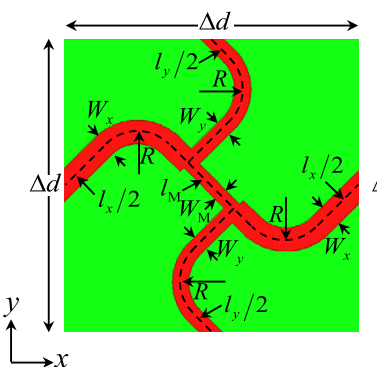

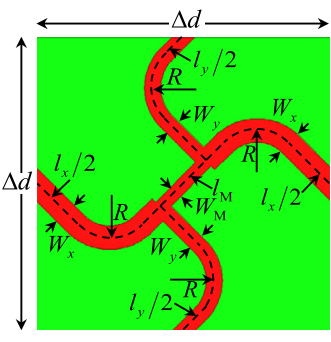

(b)
Fig. 16. Implemented unit cells of the distributed full-tensor anisotropic medium of Figure 3. (a) $\mu_{x y}>0$. (b) $\mu_{x y}<0$.

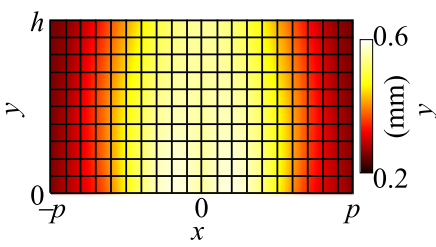

(a)

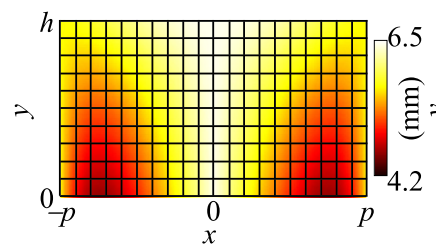

(c)

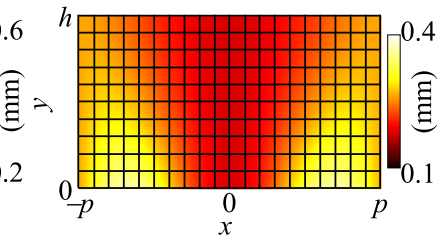

(b)
Fig. 17. Physical dimensions of the anisotropic unit cell elements for the implementation of the groove illusion medium. (a) Line width $W_{x}$. (b) Line width $W_{y}$. (c) Line length $l_{x}=l_{y}=l$. (d) Line length $l_{\mathrm{M}}$.

according to RCS calculation, which is consistent with the measured field distributions in Figure 20.

Figure 22a and b shows measured complex electric near-field distributions at a much lower frequency of $2.60 \mathrm{GHz}\left(\lambda_{g}=10.8 \Delta d\right)$ where a good impedance matching is obtained at the excitation source. It is also seen from

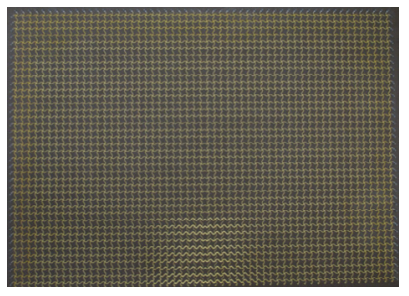

(a)

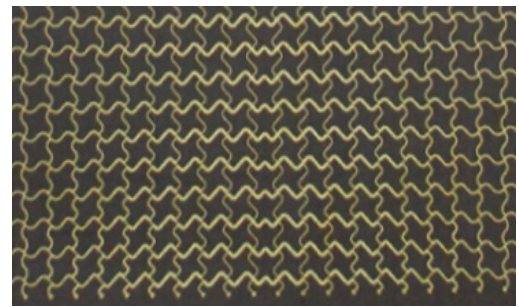

(c)

Fig. 18. Prototypes for the measurements. (a) Sample for the groove illusion. (b) Sample with the original groove. (c) Magnified picture of the coordinate transformed area.

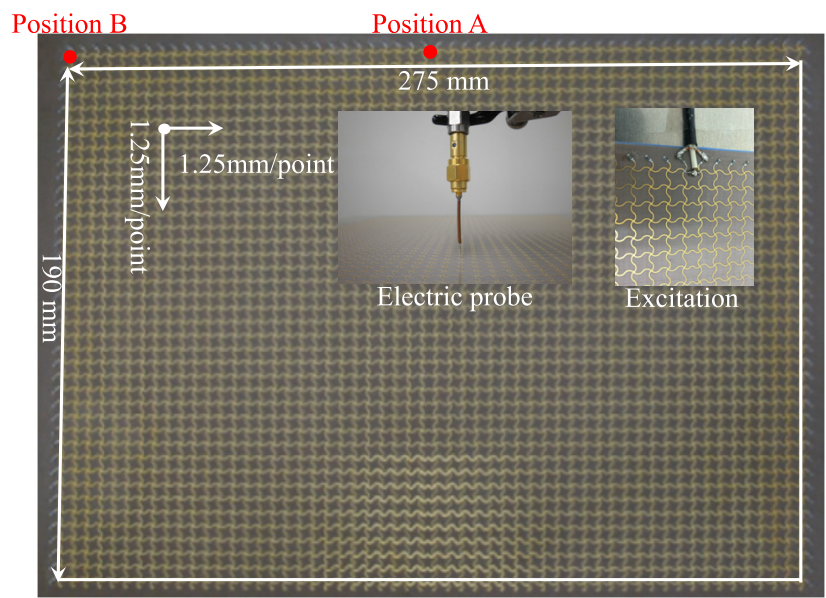

Fig. 19. Measured region. An excitation source is connected either at the position A or B. The total number of the measured points is $221 \times 153$. 

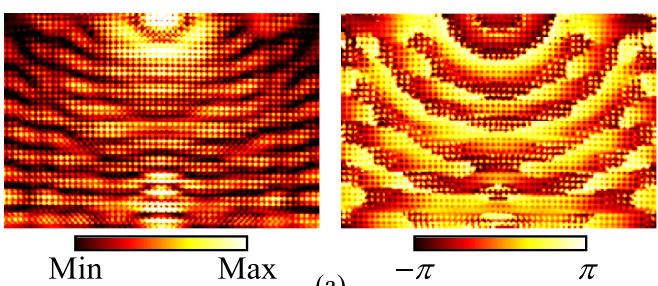

(a)
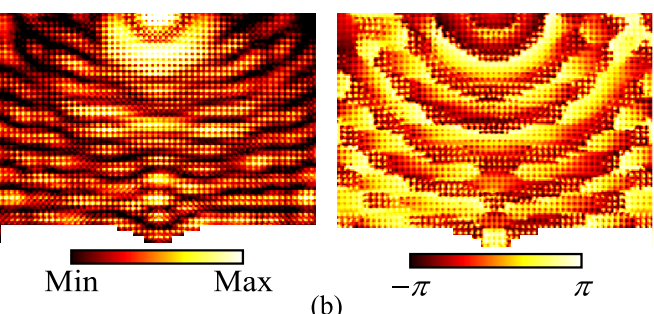

(b)

Fig. 20. Measured complex electric near-field distributions with a point source at the top center (Position A in Fig. 19) at 4.65 GHz. Left: Amplitude. Right: Phase. (a) With the groove illusion medium. (b) With the original groove.
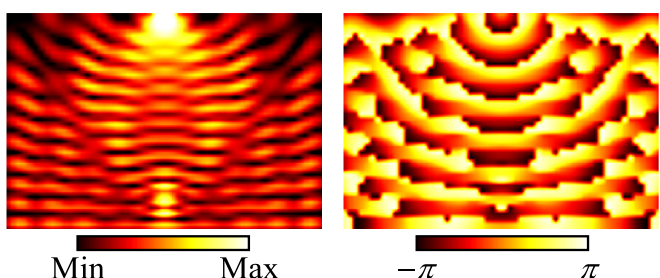

(a)
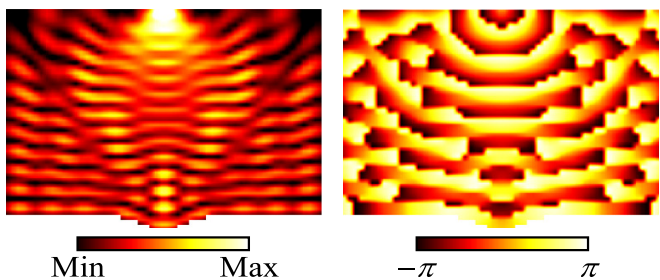

(b)

Fig. 21. Calculated complex voltage distributions corresponding to measured results in Figure 20. Left: Amplitude. Right: Phase. (a) With the groove illusion medium. (b) With the original groove.

the figure that scattered waves by the groove are well mimicked by the illusion medium. Figure $23 \mathrm{a}$ and b shows corresponding simulated results with the groove illusion medium and with the original groove, respectively, at the same frequency. By comparing Figure 22 with 23, it is seen that the measured field distributions agree well with the simulated results. According to RCS calculation, major scattered waves are in 0 and $\pm 48 \mathrm{deg}$ in this case. From these results, the broadband characteristics of the illusion medium from $2.60 \mathrm{GHz}$ to $4.65 \mathrm{GHz}$ are confirmed.
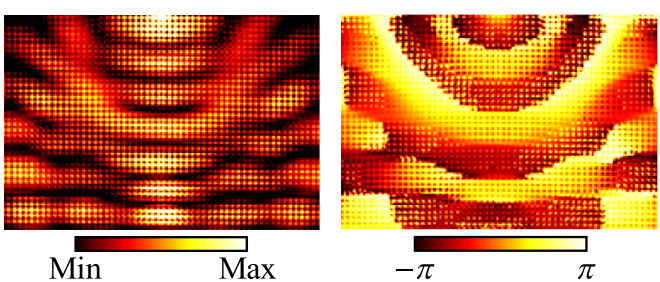

(a)
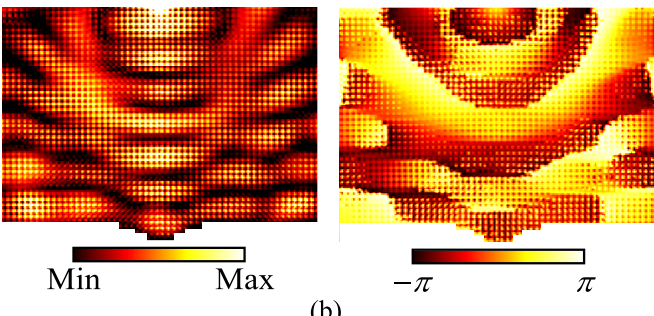

(b)

Fig. 22. Measured complex electric near-field distributions with a point source at the top center (Position A in Fig. 19) at 2.60 GHz. Left: Amplitude. Right: Phase. (a) With the groove illusion medium. (b) With the original groove.
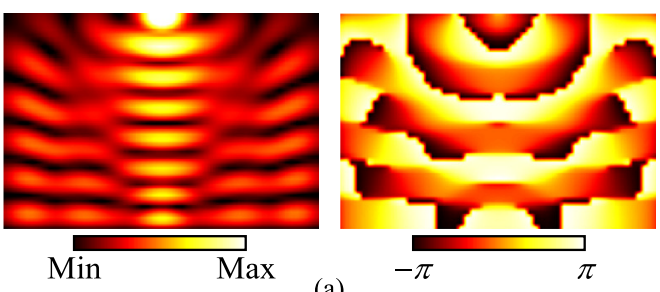

(a)
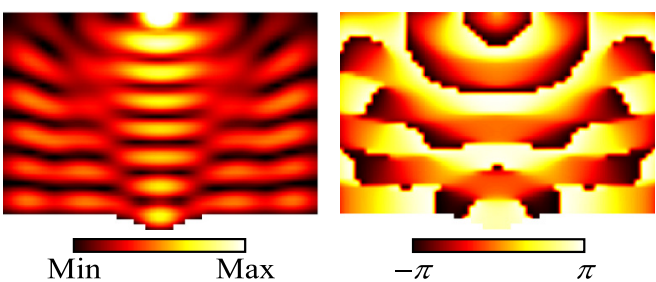

(b)

Fig. 23. Calculated complex voltage distributions corresponding to measured results in Figure 22. Left: Amplitude. Right: Phase. (a) With the groove illusion medium. (b) With the original groove.

\subsubsection{Field distributions with a source at the top corner}

Next, the excitation position is moved to the top corner (Position B in Fig. 19) and similar near-field measurements are carried out. Figure 24a and b shows measured complex electric near-field distributions at $4.65 \mathrm{GHz}$ for the cases with the groove illusion medium and with the original groove, respectively. It is seen from Figure 24 that the field distributions agree well with each other as well. Figure 25 shows corresponding simulated results to Figure 24. 

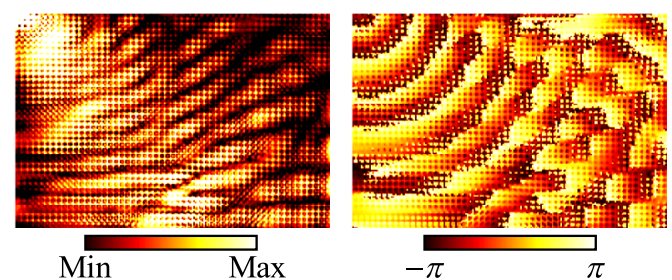

(a)
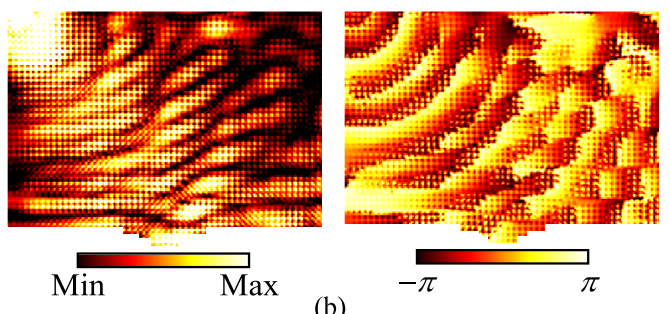

(b)

Fig. 24. Measured complex electric near-field distributions with a point source at the top corner (Position B in Fig. 19) at 4.65 GHz. Left: Amplitude. Right: Phase. (a) With the groove illusion medium. (b) With the original groove.
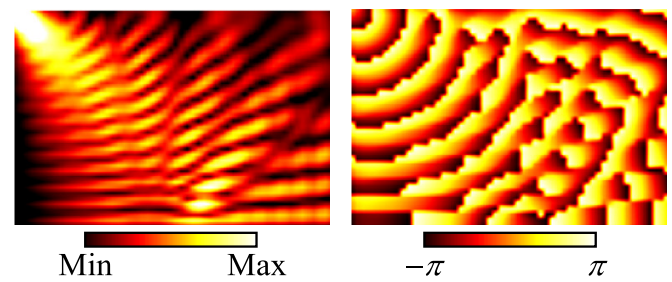

(a)
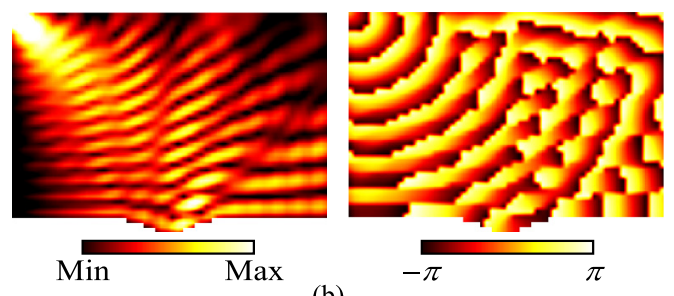

(b)

Fig. 25. Calculated complex voltage distributions corresponding to measured results in Figure 24. Left: Amplitude. Right: Phase. (a) With the groove illusion medium. (b) With the original groove.

According to RCS calculation, simulated major scattered waves are in $+35,+12$, and -9 deg in this case, which is also consistent with the measured field distributions in Figure 24. Figure 26a and b shows measured complex electric near-field distributions at $2.60 \mathrm{GHz}$ for the cases with the groove illusion medium and with the original groove, respectively. It is seen from Figure 26 that the field distributions agree well with each other also in this case. Figure 27 shows corresponding simulated results to Figure 26. According to RCS calculation, simulated major scattered waves are in +32 and $-7 \mathrm{deg}$, which is also consistent with the measured field distributions in Figure 26. Thus, it is concluded that the angle independent
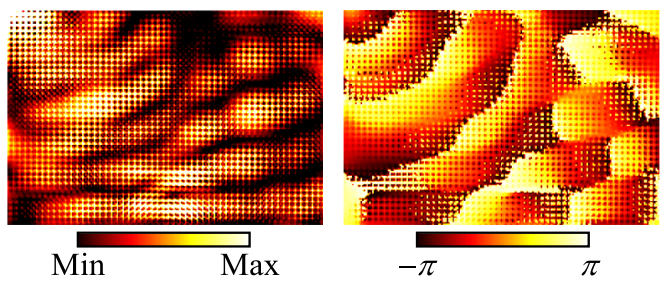

(a)
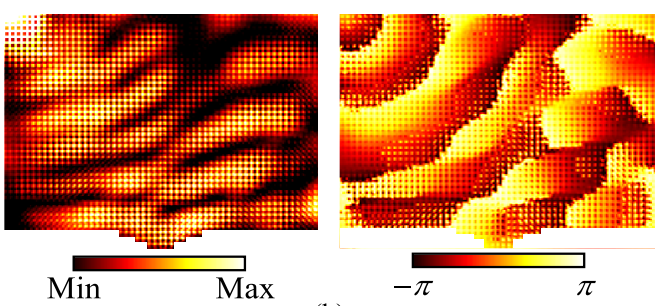

(b)

Fig. 26. Measured complex electric near-field distributions with a point source at the top corner (Position B in Fig. 19) at 2.60 GHz. Left: Amplitude. Right: Phase. (a) With the groove illusion medium. (b) With the original groove.
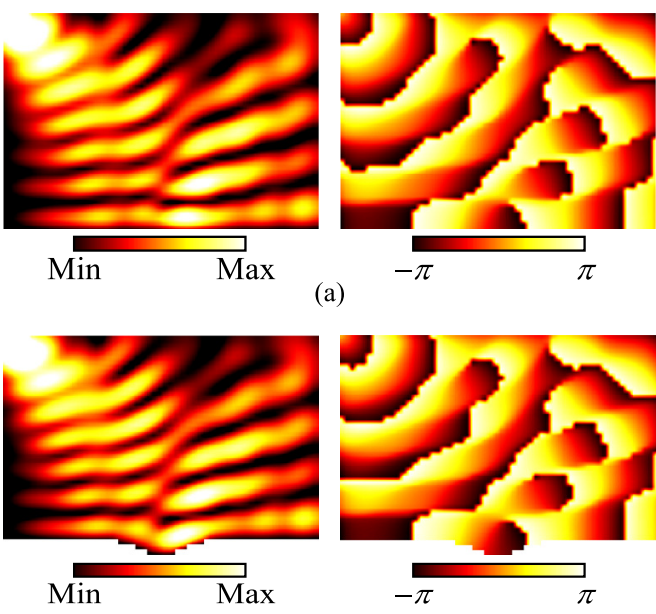

(b)

Fig. 27. Calculated complex voltage distributions corresponding to measured results in Figure 26. Left: Amplitude. Right: Phase. (a) With the groove illusion medium. (b) With the original groove.

and broadband operations of the groove illusion medium are confirmed experimentally.

\section{Conclusions}

Broadband transmission-line illusions based on transformation electromagnetics have been demonstrated by using the distributed full-tensor anisotropic medium. First, the concepts of a groove and a bump illusion media mimicking scattered waves by a groove and a bump have been presented based on transformation electromagnetics, and the design formulas of the distributed full-tensor aniso- 
tropic medium have been shown. Then, the groove and bump illusion media have been designed with the transmission-line anisotropic medium. The broadband and angle independent operations have been confirmed by calculating the scattered field distributions and the RCSs with SPICE circuit simulations and the validity of the design theory has been shown. In addition, the groove illusion medium has been implemented on a dielectric substrate with microstrip-line technology. Near-field measurements have been carried out for different two source positions and it has been confirmed that the groove illusion medium well mimics scattered waves by the original groove in the broadband frequency range as wide as from $2.60 \mathrm{GHz}$ to $4.65 \mathrm{GHz}$. It is noted that the groove illusion medium operates fundamentally at any lower frequencies down to DC.

Incidentally, we can implement the illusion medium for a plane wave illumination by stacking multiple transmission-line structures. In this case, some design modifications are required for the plane wave to transmission-line mode conversion and the impedance matching with a free space.

\section{References}

1. T. Nagayama, A. Sanada, Planar distributed full-tensor anisotropic metamaterials for transformation electromagnetics, IEEE Trans. Microw. Theory Techn. 63, 3851-3861 (2015)

2. J. Li, J.B. Pendry, Hiding under the carpet: A new strategy for cloaking, Phys. Rev. Lett. 101, 203901 (2008)

3. P. Alitalo, F. Bongard, J-F. Zürcher, J. Mosig, S. Tretyakov, Experimental verification of broadband cloaking using a volumetric cloak composed of periodically stacked cylindrical transmission-line networks, Appl. Phys. Lett. 94, 014103 (2009)

4. R. Liu, C. Ji, J.J. Mock, J.Y. Chin, T.J. Cui, D.R. Smith, Broadband ground-plane cloak, Science 323, 366-369 (2009)

5. J. Valentine, J. Li, T. Zentgraf, G. Bartal, X. Zhang, An optical cloak made of dielectrics, Nat. Mater. 8, 568-571 (2009)

6. J.H. Lee, J. Blair, V.A. Tamma, Q. Wu, S.J. Rhee, C.J. Summers, W. Park, Direct visualization of optical frequency invisibility cloak based on silicon nanorod array, Opt. Express 17, 12922-12928 (2009)
7. L.H. Gabrielli, J. Cardenas, C.B. Poitras, M. Lipson, Silicon nanostructure cloak operating at optical frequencies, Nat. Photonics 3, 461-463 (2009)

8. S. Tretyakov, P. Alitalo, O. Luukkonen, C. Simovski, Broadband electromagnetic cloaking of long cylindrical objects, Phys. Rev. Lett. 103, 103905 (2009)

9. X. Liu, C. Li, K. Yao, X. Meng, W. Feng, B. Wu, F. Li, Experimental verification of broadband invisibility using a cloak based on inductor-capacitor networks, Appl. Phys. Lett. 95, 191107 (2009)

10. B. Kanté, D. Germain, A. de Lustrac, Experimental demonstration of a nonmagnetic metamaterial cloak at microwave frequencies, Phys. Rev. B 80, 201104 (2009)

11. T. Ergin, N. Stenger, P. Brenner, J.B. Pendry, M. Wegener, Three-dimensional invisibility cloak at optical wavelengths, Science 328, 337-339 (2010)

12. M. Zedler, G.V. Eleftheriades, 2D transformation optics using anisotropic transmission-line metamaterials, in IEEE MTT-S Int. Microw. Symp. Dig. 33-36 (2010)

13. H.F. Ma, T.J. Cui, Three-dimensional broadband ground-plane cloak made of metamaterials, Nat. Commun. 1, 21 (2011)

14. D. Shin, Y. Urzhumov, Y. Jung, G. Kang, S. Baek, M. Choi, H. Park, K. Kim, D.R. Smith, Broadband electromagnetic cloaking with smart metamaterials, Nat. Commun. 3, 1213 (2012)

15. J.B. Pendry, D. Schurig, D.R. Smith, Controlling electromagnetic fields, Science 312, 1780-1782 (2006)

16. D. Schurig, J.B. Pendry, D.R. Smith, Calculation of material properties and ray tracing in transformation media, Opt. Exp. 14, 9794-9804 (2006)

17. D. Schurig, J.J. Mock, B.J. Justice, S.A. Cummer, J.B. Pendry, A.F. Starr, D.R. Smith, Metamaterial electromagnetic cloak at microwave frequencies, Science 314, 977-980 (2006)

18. T. Nagayama, A. Sanada, Coordinate transformation resonators, Appl. Phys. Lett. 112, 234101 (2018)

19. Y. Lai, J. Ng, H.Y. Chen, D.Z. Han, J.J. Xiao, Z.Q. Zhang, C.T. Chan, Illusion Optics: The optical transformation of an object into another object, Phys. Rev. Lett. 102, 253902 (2009)

20. T. Nagayama, A. Sanada, Illusion medium mimicking scattered waves of a bump on a flat surface based on transformation electromagnetics, in The 5th Advanced Lasers and Photon Sources, 1-2 (2016) 